\title{
Negotiating Agency and Control: \\ Theorizing Human-Machine Communication From a Structurational Perspective
}

\author{
Jennifer L. Gibbs ${ }^{1}\left(\mathbb{D},{\text { Gavin L. } \text { Kirkwood }^{1}\left(\mathbb{D}, \text { Chengyu Fang }^{1}(\mathbb{D} \text {, and J. Nan Wilkenfeld }\right.}^{1}\right.$ (D) \\ 1 Department of Communication, University of California, Santa Barbara, CA, USA
}

\section{Abstract}

Intelligent technologies have the potential to transform organizations and organizing processes. In particular, they are unique from prior organizational technologies in that they reposition technology as agent rather than a tool or object of use. Scholars studying human-machine communication (HMC) have begun to theorize the dual role played by human and machine agency, but they have focused primarily on the individual level. Drawing on Structuration Theory (Giddens, 1984), we propose a theoretical framework to explain agency in HMC as a process involving the negotiation of control between human and machine agents. This article contributes to HMC scholarship by offering a framework and research agenda to guide future theory-building and research on the use of intelligent technologies in organizational contexts.

Keywords: intelligent technologies, human-machine communication, structuration, agency, control

An earlier version of this manuscript was presented at the ICA conference in Washington, DC, in May 2019.

CONTACT Jennifer L. Gibbs (1) - Department of Communication • University of California • Santa Barbara, CA 93111, USA • gibbs@comm.ucsb.edu 


\section{Introduction}

Intelligent technologies, a class of emerging technologies fueled by artificial intelligence (AI), have great potential to transform organizations and organizing processes (Bailey \& Barley, 2020). Such technologies, including machine learning, robotics, and smart sensors, are programmed to mimic human capacity to learn from various inputs (i.e., new data and pattern recognition) and to respond to stimuli modeling human responses (West \& Allen, 2018). From intelligent personal assistants, such as Siri and Alexa, to algorithmic systems such as Uber's ridesharing application, AI is increasingly ubiquitous across industries with the potential to generate annual global value of over $\$ 5$ trillion (Chui \& Malhotra, 2018). The use of intelligent technologies in organizations is also growing, and whether embodied in a device or running in the background as algorithms, they are taking on increasingly agentic roles. For instance, in 2016 the Finnish company Tieto appointed an AI agent, Alicia T., as a member of the leadership team of one of its business units (Diktonius, 2016). Similarly, IBM's Watson Talent aids and plays several critical roles for both employees and employers in human resource processes (IBM Watson Talent, 2021). In these cases, intelligent technologies differ from prior information and communication technologies (ICTs) in that they have the potential to take on a more active role and work collaboratively with humans (Chui \& Malhotra, 2018).

Prior research on technology-mediated work (e.g., Raghuram et al., 2019) has primarily conceptualized technology as object or tool of use; however, the growing use of AI for decision-making and other collaborative functions repositions technology as agent through the notion that it can serve as a social agent (Banks \& de Graaf, 2020; Gunkel, 2012; Guzman, 2020). This notion is not new, but dates back to the Computers as Social Actors paradigm (CASA) (Gambino et al., 2020; Reeves \& Nass, 1996), which posits that humans can treat machines with social potential as social actors, respond to them emotionally, and identify with them as teammates or colleagues. While the CASA paradigm is well poised to explain individual level interactions with computers (Gaudiello et al., 2016; Sparrow et al., 2011; Waytz et al., 2014), it offers limited insights into processes of human-machine communication (HMC) at the group or organizational level. Intelligent technologies challenge our notions of communication to balance both anthropocentric but technocentric perspectives on agency (Banks \& de Graaf, 2020) as well as raising concerns about algorithmic control, a new form of rational control that is facilitated by algorithmic systems (Kellogg et al., 2020).

To better theorize these shifts, we draw on Structuration Theory (ST; Giddens, 1984) to develop a theoretical framework that helps to explain the relationship between human and machine agency as well as how both are implicated in a dialectic of control. Our goal here is not to offer specific propositions or testable hypotheses, but rather to leverage ST as a sensitizing device to help researchers theorize issues of agency, structure, and control in human and machine relationships (Pozzebon \& Pinsonneault, 2005). Through the discussion of related concepts and illustrative examples, this paper contributes to HMC scholarship by offering a theoretical framework and research agenda to guide future theory-building and research on the use of intelligent technologies in organizational contexts. We also extend ST by extending the notion of agency to encompass both human and machine agency in a dialectic of control. 


\section{Structuration Theory: The Duality of Structure}

An essential question for scholars studying how humans interact with increasingly intelligent technologies is what their relationship might look like. Inevitably, scholars have turned toward concepts such as agency and control to understand HMC. For instance, recent work shows the necessity to carefully consider the amount of agency that intelligent technologies have in relation to their human collaborators as machines become increasingly perceived as having agency (Fraune, 2020; Guzman \& Lewis, 2020). As Banks \& de Graaf (2020) point out, we should think about "human and machine roles in communication processes in a more egalitarian fashion" (p. 19) where the agency, interactivity, and mutual influence of both humans and machines are taken into account. An emerging recognition is that HMC is relational in nature and is constituted in interactions between humans and machines (Guzman, 2018; Suchman, 2007). For instance, Suchman takes a critical anthropological perspective on human-machine interaction, arguing that the boundaries between humans and machines are discursively and materially enacted, and that as such they can be reconfigured through an interactive process. However, aside from a few notable exceptions, such as the field of automated journalism (Lewis et al., 2019), the notion of HMC as an organizational process has not yet been adequately theorized. Indeed, the lack of attention to HMC within the field of organizational communication is evident in the fact that over the past 5 years (2016-2020), only two (less than 1\%) of the 204 paper sessions sponsored by the Organizational Communication Divisions of both the National Communication Association and the International Communication Association were related to intelligent technologies or artificial intelligence.

This motivates us to draw on structuration theory to explain the process through which agency and control are negotiated in HMC. At its theoretical roots, ST (Giddens, 1979; 1984) explains the structure-agency debate that constitutes a central theme in social theory broadly and in communication theory more specifically (Banks \& Riley, 1993; Riley, 1983). According to Giddens (1984), structure and agency are recursively related in that humans create rules and resources-or structures-that in turn enable and constrain their actions and behavior. Agents and structures are not independent but are mutually constituted through the duality of structure, as "the structural properties of social systems are both medium and outcome of the practices they recursively organize" (Giddens, 1984, p. 25). In this perspective, social actors both draw upon and reproduce structural features of wider social systems in their day-to-day activities. Although structure is more enduring, the social systems in which it is instantiated are comprised of situated activities of human agents that are reproduced across time and space. While human agents have the ability to act knowledgeably and reflexively, the activities that lead to reproduction of social and institutionalized practices often have unintended consequences; this helps to explain both how social systems endure as well as how they change and evolve.

While ST has traditionally focused on social systems and structures, organizational scholars have drawn attention to technological systems and structures as well. Previous research on the structuring role of technology in organizations has highlighted the ways people make sense of, appropriate, and develop practices around technology (Leonardi, 2011; Majchrzak et al., 2000). Scholars have applied ST to understand the recursive relationship between human agency and sociotechnological structures (Barley, 1986; 
DeSanctis \& Poole, 1994; Orlikowski \& Yates, 1994; Stillman \& Stoecker, 2005). For example, Barley's (1986) account of the implementation of computerized tomography (CT) scanners at two hospitals illustrated that adoption of the new CT machines not only restructured the work practices of the radiologists and CT technologists, but also disrupted and redefined occupational roles and power relationships between these two groups of experts. Orlikowski and Yates (1994) found that knowledge workers developed rules around email use by drawing on established genres and genre repertoires that served to structure their communicative practices, which were rich and varied and changed over time. DeSanctis and Poole developed Adaptive Structuration Theory (AST) to explain how group members appropriate the structures provided by technology, often in combination with other social and organizational structures, in ways that may be either faithful to or at odds with the initial intent of the designers. This line of research helps to explain the recursive relationship between organizing and technology, and the way in which this process evolves over time. However, it treats agency as confined to human actors and structure as confined to technology (which is regarded as object rather than actor). Intelligent technologies require an extension of structurational theory to account for both human and machine agency and the ways they are instantiated and negotiated in HMC.

\section{A Structurational View of Human-Machine Communication}

Traditionally, humans are the only type of organizational actor that meets the criteria for agency in ST (Giddens, 1979; McPhee et al., 2014). According to ST, agency stems from one's capacity to exercise power in meaningful or normative ways and to reflexively monitor one's actions, which allows them to understand how their actions either reify or transform the structures in which they act (Giddens, 1984). The rapid advancement of intelligent technologies brings about the need to expand ST by contending with both human and machine agency.

\section{Human Agency and Machine Agency}

Communication scholars and social theorists more broadly have defined agency as a capacity or ability that originates from the resources, rights, and obligations that an individual social actor owns and occupies (Abdelnour et al., 2017; DeSanctis \& Poole, 1994; Giddens, 1979). As Emirbayer and Mische (1998) pointed out, an agent should be able to develop habits from past experience, make judgments about their present environment, and imagine their future actions, which they refer to as the "Chordal Triad." Giddens defines agency as the ability to make a difference, or the "ability to do otherwise" (Giddens, 1984). While it is important to recognize the agentic capacity of intelligent technologies, our focus here is not to redefine agency. Thus, in this paper, we follow previous discussions and define agency colloquially as one's capacity to reflect, adapt, and act.

The idea of technology and material artifacts having agency is not new. Scholars studying the relationship between humans and technology have increasingly recognized that physical objects can also have agency as we inscribe behaviors into them and give them the capacity to mediate our interactions (Latour, 1991) and as we interact with physical objects and technologies through various work arrangements in organizations (Orlikowski, 2007; 
Orlikowski \& Scott, 2008). In this way, social and material agencies are mutually imbricated (Leonardi, 2011). More importantly, recent theorizing about digital agency of AI (Ågerfalk, 2020) and symbiotic agency in chatbots (Neff \& Nagy, 2016) has begun to recognize that intelligent technologies themselves have increasingly more agency as a result of our programming and design. A distinctive feature of intelligent technologies is their ability to perform tasks autonomously, taking over control of organizational tasks such as scheduling meetings, screening job candidates, or even making recommendations for social relationship development (Perc et al., 2019; Russell \& Norvig, 2019). Technologies such as, for example, Google's page ranking algorithm, have the capacity to act symbolically (i.e., provide recommendations and rank search results) on behalf of the individuals and organizations who developed them. Essentially, machine agency refers to the capacity of intelligent technologies to perform specific tasks given its material features. Similar recognition that machines might differ in their levels of agency can also be found in early work on systems theory (Boulding, 1956; Pondy \& Mitroff, 1979). Systems may vary in levels of agency from reactive systems (which respond to environmental cues in predetermined ways) to goal-driven systems (which incorporate goals set by others) and problem-solving systems (which are more complex as they are capable of solving problems in achieving their goals and adjusting goals and routines). At even higher levels of agency are self-aware systems (which are aware of themselves and other agents and can reflect on their own activity) and multivocal systems (composed of multiple selves, can orient differently to different parts of the situation, and may reconstitute themselves through problem-solving and reflection). These levels of agency can be applied to various AI tools and systems. Therefore, AI may differ in terms of how responsive it is to external and internal cues, its freedom of action and reflexivity to monitor action, its decision-making ability, and its awareness of other agents and of its own agency.

Knowledgeability. Giddens identified two criteria for agency. The first is knowledgeability, the tacit information or discursive resources that actors have about the circumstances that can inform their future actions. In Giddens's (1984) words, knowledgeability is about "what agents know about what they do, and why they do it" (p. xxiii). Taking comprehensive data processing as an example, intelligent technologies that have the ability to collect a variety of data on employees including biometrics, messages between users, and the location of workers can be considered as knowledgeable about their collaborators in organizations (Kellogg et al., 2020). For example, Duhigg (2012) reported on a case where the retail corporation Target used machine learning and pattern recognition of consumer purchase data (including changes in buying behavior of body lotions and cleaning products) to predict pregnancy in its female shoppers-sometimes even before they or their families knew they were pregnant. The AI technology that Target used in this case developed predictions based on the information that it had about its customers. It acted knowledgeably as a tool for organizations. In the rideshare context, Uber's AI can leverage multiple data points to manage employees including customer ratings, geographic location, how cooperative drivers are with accepting assignments, and how long rides take (Lee et al., 2017; Rosenblat \& Stark, 2016). In contrast to the Target example, AI in this case acted in a managerial capacity through directing and facilitating interactions between Uber employees and their customers. Chan and Humphreys (2018) demonstrated that the data collected on Uber drivers 
allows AI technologies to surveil its contracted workforce on behalf of the organization by tracking spatial movements and customer interactions. These examples illustrate that current intelligent technologies know what they should do, even though they do not yet know why.

When developing ST, Giddens (1984) recognized that the knowledgeability of human actors will always be limited in some ways and we argue that this is also true of intelligent technologies. Levels of awareness are likely to vary between three different types of consciousness that inform actor knowledgeability: discursive consciousness, practical consciousness, and unconsciousness. Discursive consciousness refers to an actor's ability to understand the purpose of their actions and explain those reasons in social interactions. In contrast to discursive consciousness, practical consciousness consists of an actor's ability to recognize how a task needs to be completed and competently act, and is more habituated or routinized and taken for granted. Lastly, Giddens (1984) acknowledged that actors often are unconscious or unaware of (a) conditions that shape actions and (b) unintended consequences of actions within larger structures. Ultimately, agency in ST is less focused on the intentions and goals of actors and instead emphasizes the patterned actions of agents (Giddens, 1979; 1984). Giddens (1984) argued that "knowledgeability is founded less upon discursive than practical consciousness" (p. 25). Although intelligent technologies are not aware of the reasons behind their actions and cannot exercise discursive consciousness, they are capable of enacting more routinized practical consciousness that directs actions in a larger structure. We argue that the lack of discursive consciousness in intelligent technologies does not prohibit ST from being applied in HMC. Rather, distinguishing which types of consciousness inform knowledgeability in intelligent technologies contributes to our understanding of machine agency and how machines may complement or hinder human agency in organizations.

Reflexive monitoring. The second criterion of agency is reflexive monitoring. This refers to the ability to monitor one's actions, evaluate the outcomes of these actions, and develop awareness of the settings and contexts around the actions (Giddens, 1984). A good example of reflexive monitoring in intelligent technologies is instantaneous processing. This refers to the high velocity of algorithmic computation and the ability to incorporate performance systems in real time (Kellogg et al., 2020). Examples of instantaneous processing include AI-enabled technologies such as Robot vacuums or autonomous vehicles that need to continuously monitor their surroundings and respond reflexively in order to function (Elliott, 2019). Admittedly, intelligent technologies used in organizations or in households are not quite yet at a stage where they can monitor their own performance and adapt future actions based on past performance, but they are certainly at the stage where they can monitor their own actions by engaging in a flow of context-responsive behavior. However, as these examples show, intelligent technologies in their current form still rely on human interventions and implementations to fully utilize their own knowledgeability and reflexive monitoring.

\section{Control and Structure}

To address the relationship between human agency and machine agency, it is important to recognize that ST conceptualizes power as relational as does Foucault (1979). According 
to Giddens (1984), power can be seen as a dialectic of control in which subordinate social actors can influence the choices and behaviors of their supervisors and create transformative outcomes by drawing on resources that they control and have access to, namely structure. In other words, structure enables knowledgeable and reflexive agents to exert power and control. Not surprisingly, control systems also play an important role in structuring and holding organizational units together by providing rules by which to direct work tasks, evaluate work, and reward and discipline workers (Edwards, 1981). The organizational communication literature has identified various forms of control that arise in organizations, ranging from more direct, top-down approaches used in hierarchical organizational structures to more indirect, peer-based, or internalized forms of concertive or unobtrusive control (Tompkins \& Cheney, 1985).

The discussion around issues of control between humans and machines is not new but is rooted in discussions of the ways in which earlier forms of technology were implicated in the negotiation of organizational control. For instance, Levy (2015) found that electronic monitoring systems exerted new forms of control over workers in the trucking industry, by providing new metrics for evaluating truckers' job performance and challenging their accounts of local and biophysical conditions, and that truckers engaged in resistance practices to subvert or circumvent these control mechanisms. Cybernetic scholars have also centered their theorizing about communication around issues of information and control (Guzman, 2016). As Wiener (1950) pointed out, "the significance of machines in human society" is reflected in their relationships to communication and control (p. 23). The shift from technology as a tool or object of use to automated machines that manage human actions is a reflection of the political and power struggles between workers and managers (Guzman, 2016; Noble, 2011). With intelligent technologies, control can be even more adaptive. As our discussion of Uber would suggest, intelligent technologies such as AI may exert even more control through the capacity to self-correct through the use of sophisticated sensors and delicate feedback systems (Chan \& Humphreys, 2018; Guzman, 2016). More importantly, the increasing amount of agency and capacity for interactivity also opens the door for peer or collaborative relationships in human-machine interactions that goes beyond the question of who has control (Janlert \& Stolterman, 2017; Sundar et al., 2016).

HMC literature also acknowledges the relational nature of the negotiation of control between humans and intelligent technologies. Malone (2018) pointed out that AI in organizational use could play differing roles and these roles range in terms of the amount of control given to AI, from low to high. For instance, intelligent technologies can not only act as an assistant when they function with some degree of human input (i.e., use voice command to instruct Google Home), but can also act as a peer if a digital assistant (e.g., chatbot) interacts with other humans on behalf of an individual (Moore, 2019). More importantly, intelligent technologies can serve in a variety of roles depending on the context and use. In other words, these roles range on a control continuum and are not static. Rather, they fluctuate depending on the experience of individuals. An example of how AI's role shifts based on the eye of the beholder is autonomous vehicles (Malone, 2018). A self-driving car may be viewed as a tool to its human passengers, as it is being used to transport them from one point to another. That same self-driving car, however, could be viewed as a peer by other drivers that share the road with the vehicle. Similarly, an AI project management system that manages the workflow of organizational teams could be viewed as having a 
managerial role in their organization by its subordinates but be seen as a peer or even assistant by human managers. Thus, similar to ST, control in HMC is dependent on the agency of machines in relation to a group of individuals who also have varying degrees of agency.

Structure can take many forms in HMC. According to Giddens (1984), the duality of structure can take three forms: domination, signification, and legitimation. Domination refers to the unequal distribution of power resources, which may be available to some agents (e.g., managers) and not others (e.g., lower-level employees) (McPhee et al., 2014). For example, in cases where companies appoint AI agents to play an official organizational role, intelligent technologies have authoritative power (over people) or allocative power (over resources) as they make decisions for organizations, recommend strategies to managers and workers, or provide work assignments to employees (Chan \& Humphreys, 2018; Diktonius, 2016; IBM Watson Talent, 2021). Similarly, the ways organizational members interpret the role that intelligent technologies play (Malone, 2018) or how society views AI ontologically (Guzman, 2020) can also provide structures for signification, the interpreted meaning through lived experience in context of the structure, or legitimation, expectations and norms that can influence the consequences of actions (Giddens, 1984).

Algorithmic control and rendition as mechanisms of structuration. We propose that structuration in HMC takes place through algorithmic control and the process of rendition. Algorithmic control is a form of technical control that is prevalent on labor platforms in the gig economy (i.e., ride sharing and the food delivery industry). Companies can use algorithms to shape and influence the behavior and outcomes of workers (Wood et al., 2019). Through the process of rendition (also known as datafication and mediatization; Chan \& Humphreys, 2018), human action is encoded into organizational or algorithmic data (Zuboff, 2019), which in turn constitute structures that enable and constrain employee behavior and outcomes. For instance, Wood et al. argue that organizations using algorithmic management in the form of platform-based rating or recommendation systems exert a form of Taylorist informational control on their employees and that computerization intensifies work by increasing monitoring, increasing the pace, and extending work activity beyond the conventional workday.

The hallmark of algorithmic systems is their ability to record and aggregate behavioral information and statistics from a variety of internal and external sources (Kellogg et al., 2020). Algorithms take on agency and are able to serve a managerial role in organizations in part due to advancements in deep machine learning through neural networks. In deep machine learning, computers use Bayesian statistical models to recognize patterns in large amounts of labeled data (often referred to as "tagged data") (Krizhevsky et al., 2012). Deep machine learning follows the computer science adage, "garbage in, garbage out" which indicates that human agency (in the form of the quality of the data labeling) will directly impact the machine's ability to learn from the data (Biggio \& Roli, 2018; Krizhevsky et al., 2012). The algorithms behind Uber's customer rating system exert influence over the behavior and work outcomes of Uber drivers, who can be automatically fired for dropping below a certain average rating-motivating them to perform emotional labor and engage in strategic behavior to increase their customer ratings (Chan \& Humphreys, 2018; Rosenblat \& Stark, 2016). Even without formal power structures (structures of domination), Uber drivers may still see the behind-the-scenes algorithm as an AI manager (structures of signification). In 
other words, algorithmic control through rendition is reflective of a structurational process of HMC. As a structurational process, human managers are utilizing machine agency that uses data collected from human actors to minimize workers' intervention and automatically generate data, assess worker behavior, and assign work tasks (Newlands, 2020). Machine agency in turn enables and constrains the decision-making and other behavior of workers and organizations.

The duality of structure is one of the fundamental sensitizing devices in the structurational approach (Giddens 1979; 1984). Machine learning processes enable algorithmic management to reflect this duality of structure as they blur the lines between human agency and organizational structures. Orlikowski (1992) argued that there is a duality of technology, or an inherently interdependent relationship between objective technological properties and the interpretive flexibility that facilitates social construction of technology use. Orlikowski (1992) recognized that technology is a product of human action because humans create and adapt the technology; however, in rendition agency and structure are even more inseparable, as user behavior actually forms the basis of the technology. Rendition also allows algorithmic managers to adapt in real-time based on changes in user behavior.

\section{Negotiating Agency and Control: Toward a Research Agenda}

Despite the growing use of intelligent technologies in organizations, HMC scholarship has focused primarily on explaining human-machine communication at the individual level, without consideration of organizational dynamics and control mechanisms. Intelligent technologies differ from prior organizational technologies in that they have the potential to reposition our conceptualization of technology as object to one of technology as agent. While the notion of computers as social agents is not new and stems from the CASA paradigm (Reeves \& Nass, 1996), CASA is more focused on explaining individual interactions with AI, rather than group and organizational level processes. Our structurational framework constitutes an important first step in conceptualizing the role agency and control play in understanding the processes of HMC in organizational context.

A key feature of the HMC structuration process is that it is dialectical, as organizational actors are constantly bound between the negotiations between human and machine agency as well as between traditional organizational control and algorithmic control. Therefore, we draw on the notion of dialectics in ST to conceptualize what this process might look like in organizational use of intelligent technologies. Giddens (1979) was one of the first scholars to conceptualize dialectics in social structures, which arise when the operation of one structural principle presupposes another that tends to undermine it (Giddens, 1984). According to Giddens (1979), dialectics or contradictions arise "in the midst of, and as a result of, the structuration of modes of system representation” (p. 141). Giddens defined the dialectic of control as a reciprocal power relationship between active agents and institutional forms of control. According to Giddens, the dialectic of control is usually constituted by conflicts around autonomy and dependence among social actors (e.g., between employees and organizations). Howard and Geist (1995) illustrated the dialectic of control in their case study on the ideological positioning of employees at a gas company in the context of a proposed merger with another company. They identified three dialectics or oppositions, between change and stability, empowerment and powerlessness, and identification 
and estrangement. Howard and Geist explained that SGC employees took either passive or active ideological positions to help them navigate the tensions with the merger. We extend the dialectic of control to examine the negotiation of power relationships between human agents and intelligent technology agents. While the dialectic of control has traditionally focused on tensions between organizational control and employee resistance, AI brings a new form of control into the mix: algorithmic control. With this structurational view in mind, we now turn to some illustrative examples in organizations to show both theoretically and practically how humans and machines negotiate this dialectic of control.

\section{Illustrative Examples}

According to Zuboff (2019), the surveillance capitalist system is characterized by technologies that accurately predict and control human behavior, and this makes the agency afforded by these technologies less prominent, hidden, or blurred. For example, car insurance premium rates are determined by a myriad of factors that do not seem to connect to driving, such as an individual's likelihood of shopping around for competitive prices based on purchasing history (O'Neil, 2016). Often, these algorithms are so complex that very few people - not even the insurance agents themselves-understand how the algorithm arrives at the premium numbers. Another example is Amazon's "Ambots," the nickname given to Amazon warehouse employees who are reviewed on the time it takes them to pick and pack items for shipment (Faraj et al., 2018). Managed by algorithms, employees are tracked in the warehouse and assigned to pick items based on the most efficient route. Employees are rewarded for improving their times or punished for having slower times. We now go into further detail on three examples to illustrate our structurational view of HMC. Each of the examples we will use- $\mathrm{A} / \mathrm{B}$ testing, humans in the loop, and automated journalismrepresent uses of intelligent technologies that are becoming more prevalent in organizations. They each reflect a dialectic of control in terms of the negotiation of human-machine agency and control.

$A / B$ testing. One example that illustrates our structurational perspective is $\mathrm{A} / \mathrm{B}$ testing. This is an example of information asymmetries in AI systems (Rosenblat, 2019), which are defined as situations "in which one party knows more than the other" (Sundararajan, 2016, p. 139). In $A / B$ testing, software designers create different versions of platforms in order to understand how differences in features impact user behavior. A/B trials can range from minor discrepancies to entirely different ecosystems for users. Rosenblat explained that in the rideshare context of Uber, different types of $\mathrm{A} / \mathrm{B}$ testing may include paying one group of employees more than another, offering different types of bonuses for giving a certain number of rides, and even different visual displays that may or may not shape work behaviors. Often, users do not know they are a part of $\mathrm{A} / \mathrm{B}$ trials as their knowledge might impact research that developers are conducting. $\mathrm{A} / \mathrm{B}$ testing enables companies to conduct more rigorous rendition, since it allows machine learning systems to observe and learn from a variety of user behavior in response to changing stimuli. In this case, machine agency is comprised mainly of reflexive monitoring, whereas the agency of users takes the form of practical consciousness as they go about their habituated work routines without awareness of how platforms are shaping their work behaviors. 
The changing nature of AI systems in A/B testing has important implications for structurational approaches to technology. One implication is that the structural rules of the technology have the potential to change from user to user; this means that there might be tensions between knowledgeability in machine and human actors. In the rideshare example, machines have more knowledge about which workers are funneled into different ecosystems, while rideshare workers may be completely unaware that there are discrepancies. The dialectic of control plays out as different features (e.g., visual displays) impact user behavior, which in turn provides data and inputs that inform, optimize, and transform the system.

Humans in the loop. A second example is humans in the loop (HIL), a phrase that describes the partnership of humans and computers (intelligent algorithms) working together on tasks to achieve the highest level of performance and efficiency (Malone, 2018). Despite the common narrative that machines will destroy jobs, the reality is that intelligent technologies can only provide a replacement for certain tasks within job roles. Companies rely on humans to perform tasks that AI cannot do or that would be inefficient or costly to develop an algorithmic system to do. Malone argued that rather than regarding intelligent technologies as replacements for humans, we should consider them as complements to human capabilities and vice versa. Workers becoming HIL will experience changes in processes and redesigning of tasks, which could be sites of struggle as the balance of control and agency becomes less equal (Brynjolfsson et al., 2018).

The collaboration process between humans and machines, also called heteromation (Ekbia \& Nardi, 2014), may vary in terms of the distribution of control and agency between humans and computers as manifested in both task distributions and the visibility of the respective agent. Collaborations involving low algorithmic control (compared to humans) include knowledge-sharing platforms such as Wikipedia, which combines human knowledge and creativity with the capabilities of the internet and data storage platform technology to build the largest encyclopedia the world has ever seen (Malone, 2018).

On the opposite end of the spectrum, collaborations involving high algorithmic control include the phenomenon known as "ghost work" (Gray \& Suri, 2019). In ghost work, humans perform tasks that an algorithm is unable to do, including cleaning and organizing unstructured data, verifying locations and identities, and creating content. In other words, rather than technology being a complement to the human, the human is the augment to the technology. Tasks done by ghost workers are typically repetitive and simple, for example, training a facial recognition algorithm to recognize a particular image by answering "yes" or "no" to whether two provided pictures are the same person (Kellogg et al., 2020). In ghost work, machine learning algorithms take on high levels of agency and algorithmic control and humans seem to disappear. For example, Microsoft's Face API uses ghost workers to increase the accuracy of identity detection for Uber's Real-time ID Check (Gray \& Suri, 2019). Uber drivers are randomly selected to provide a selfie for identity verification, and if the algorithm is unable to determine whether the profile and selfie images match, the images are sent to a ghost worker who has approximately 30 seconds to compare the pictures and decide whether they are a match or not. This human worker is critical to the system for providing training to the algorithm, safety to the Uber passenger, and continued income for the driver. This human, however, remains nameless and is only alluded to in 
passing in Uber's description of the verification process; the human gets placed into the technological black box. It is important to note that even in such cases where algorithms seem to have most of the control, humans play an important (albeit invisible) role in performing tasks designed to correct and teach the machine. This suggests that despite the increased potential for algorithmic agency and control in organizations, humans will still maintain control over tasks where the algorithm falls short. This reflects Giddens's (1984) notion that even workers in low power positions can exert agency.

Automated journalism. A third example in which algorithms are impacting agency and structural norms is automated (or algorithmic) journalism. Public scrutiny about journalistic integrity, political bias, and elitism has fueled the proliferation of algorithms in this field as they are viewed as being less biased than their human counterparts (Graefe et al., 2018). Rather than having news content selected by editors and feedback mainly consisting of paper or magazine sales, real-time analytics provide journalists and outlets instant feedback on readership and actions such as link sharing through social media. Journalists are now accountable to their readers more than their editors, and content is driven by what authors think will get the most attention (Christin, 2017). In the new field of automated journalism, news outlets such as the Associated Press are utilizing "robot authors" to create content faster and in a less costly manner than human reporters (Graefe et al., 2018). This increased use of algorithms has prompted discussions within the field on redefining previously held understanding of what it means to be an author and a journalist, as well as larger discussions on what journalism is and how the field should operate (Carlson, 2015). The phenomenon of automated journalism can be reinterpreted through a structurational lens. For instance, a study of the code and algorithms contained in mobile news apps challenges the common notion that algorithms are replacing humans as producers of news, by highlighting the role of code in helping journalists order and communicate the news (Weber \& Kosterich, 2018). This study illustrates the dialectic of control between human and machine agency, as human actors imbue decisions and agency into the code such that it takes on agency of its own and begins to occupy an editorial role, prioritizing and sorting news based on the human input it receives.

\section{Future Research Agenda and Conclusion}

Viewing HMC through a structurational framework helps to advance HMC scholarship. First, it helps to explain the recursive relationship between human and machine agency and the process through which they are mutually constituted in organizations. This structurational process occurs as human agency creates the behavior that constitutes the knowledgeability (or intelligence) of machine agency. Machines then learn by surveilling human actions, and when algorithmic systems have high predictive value, they can in turn limit human agency without people being aware of it. The process of rendition also makes it difficult for humans to oversee how machines are learning, which means increased potential for exacerbating existing biases. Second, a structurational framework provides an important counterpoint to scholarship on algorithmic control, which has emphasized its constraining structural elements (e.g., Newlands, 2020; Wood et al., 2019; Zuboff, 2019) by bringing in agency (as an interplay between human and machine agency). This highlights the fact that, 
while agentic, algorithmic systems are not a form of "autonomous technology" (Sturken et al., 2004) that exists outside of human control. Rather, they are continually produced and reproduced through human action. While they may have unintended consequences, they are continually evolving in a recursive relationship with the human actors that interact with them and provide them with inputs.

Viewing these changes through a structurational lens enables scholars to provide a broader picture not only for fellow researchers, but for practitioners as intelligent technologies impact work teams and shape organizational cultures. For example, increasing use of these technologies can affect learning and reshape what is considered expertise, which in turn can have a ripple effect as newer employees are onboarded and tensions between older and newer practices arise. In some cases, employees would resort to deviant methods to learn skills after the implementation of a new technology, called "shadow learning" (Beane, 2019). Additionally, intelligent technologies with more control over decision-making, such as intelligent personal assistants or even intelligent project managers, can interrupt team learning and knowledge sharing which can then impact overall team performance (Yan et al., 2020).

There are several avenues that future research on this topic should consider. First, there may be additional dimensions besides control and agency that impact the role of AI in collaborative practices. For instance, the degree of visibility and embodiment of intelligent technologies may influence the role they can play and the level of control they have. Future research should also consider how issues of control and agency may vary across different types of work and occupational cultures. For instance, higher skilled professionals that have deeply engrained tacit knowledge, domain-specific training, and professional hierarchies such as lawyers may have more difficulty working alongside intelligent technologies. Certain occupational practices, such as attorney-client privilege and liability, may result in certain organizational members being more resistant to using AI due to privacy or ethical concerns, concerns about its judgment, or concerns about its accuracy. There may also be different occupational cultures around blue-collar/manufacturing work versus white-collar information or knowledge work that shape perceptions and usage of intelligent technologies. These cultural differences may shape dominant discourses around automation and job security, concerns or fears about loss of control, and resistance to technology. Applying our structurational perspective to study occupational culture, for example, would involve immersive observation or interviews to learn about the particular occupational context and how institutional logics associated with it shape how intelligent technologies are used and perceived. It would also be best served through a longitudinal design to allow for observation of recursive relationship between human and machine agency and the ways in which they interact and shape one another over time.

Our structurational framework suggests new research questions to inform the HMC research agenda going forward. What will happen as intelligent technologies develop more knowledgeability and reflexive monitoring? How do organizations work with AI that can take on fully agentic roles? How can we understand new relationships between agency and structure in the rendition process? How do workers communicatively resist algorithmic control? One example of this is adversarial machine learning, in which humans intentionally provide inputs to subvert rather than improve the accuracy of AI systems (e.g., by tricking facial recognition systems to recognize a turtle as a rifle) (Biggio \& Roli, 2018). Finally, 
does algorithmic management inevitably represent a return to Taylorist control, or can it be restructured to provide more worker empowerment?

In conclusion, as intelligent technologies become more ubiquitous in organizations, it is important to understand how these new organizational agents will affect processes of organizing. Our structurational framework helps to theorize the process by which human and machine agency is mutually constituted and negotiated in a dialectic of control. One consequence of this imbrication of human and machine agency is that machines are starting to act more human at the same time as humans are starting to act more like machines. We encourage researchers to find novel ways to apply our structurational framework to help guide our understanding of $\mathrm{AI}$ and its role in organizing processes.

\section{Author Biographies}

Jennifer L. Gibbs (PhD, University of Southern California) is a Professor of Communication at the University of California, Santa Barbara as well as Co-Editor of Communication Research. Her research focuses on collaboration in global teams and other distributed work arrangements as well as the affordances of digital technologies for strategic communication practices. Her work has been published in leading journals from a variety of disciplines including Administrative Science Quarterly, American Behavioral Scientist, Communication Research, Computers in Human Behavior, Human Relations, The Information Society, Journal of Computer-Mediated Communication, Journal of Social \& Personal Relationships, Management Communication Quarterly, and Organization Science.

\section{https://orcid.org/0000-0003-1346-2960}

Gavin L. Kirkwood (MA, Western Kentucky University) is a PhD candidate in the Department of Communication at University of California, Santa Barbara. His research centers on the social impacts of emerging technologies including algorithmic managers, AIenabled facial recognition, and exoskeletons. Gavin has taught a variety of classes including small group communication, communication theory, and research methods. His work can be found in publications including Communication Studies as well as Communication and Social Media.

\section{https://orcid.org/0000-0002-5569-9782}

Chengyu Fang (MA, University of Illinois at Urbana-Champaign) is a PhD student in the Department of Communication at the University of California, Santa Barbara. His research focuses on two interconnected topics: (1) intelligent technologies and work, and (2) the emergence of esports and teams. Chengyu has taught several organizational communication and introductory communication classes. His work has been presented in national and international conferences and has been published in journals such as Communication Research and Academy of Management Proceedings.

https://orcid.org/0000-0001-7024-0243 
J. Nan Wilkenfeld (MA and MBA, UNC Charlotte) is a doctoral student in the Department of Communication at the University of California, Santa Barbara. Her research interests lie at the intersection of technology, communication, and organizing. She is currently working on a collaborative project with UCSB and Virginia Tech that is developing exoskeleton technology for industrial contexts. Before starting her $\mathrm{PhD}$, Nan worked as a freelance consultant helping small businesses and teams with leadership and team development, and organizational change. Some of her previous clients include Sterling Events, Lanikila Health and Wellness, Kinetic Heights, and Atrium Health.

https://orcid.org/0000-0002-1591-1910

\section{References}

Abdelnour, S., Hasselbladh, H., \& Kallinikos, J. (2017). Agency and institutionsin organization studies. Organization Studies, 38, 1775-1792. https://doi.org/10.1177/0170840617708007 Ågerfalk, P. J. (2020). Artificial intelligence as digital agency. European Journal of Information Systems, 29(1), 1-8. https://doi.org/10.1080/0960085X.2020.1721947

Bailey, D. E., \& Barley, S. R. (2020). Beyond design and use: How scholars should study intelligent technologies. Information and Organization, 30, 100286. https://doi.org/10.1016/j. infoandorg.2019.100286

Banks, J., \& de Graaf, M. (2020). Toward an agent-agnostic transmission model: Synthesizing anthropocentric and technocentric paradigms in communication. HumanMachine Communication, 1, 19-36. https://doi.org/10.30658/hmc.1.2

Banks, S. P., \& Riley, P. (1993). Structuration theory as an ontology for communication research. Communication Yearbook 16, 167-196. https://doi.org/10.1080/23808985.199 3.11678851

Barley, S. (1986). Technology as an occasion for structuring: Evidence from observations of CT scanners and the social order of radiology departments. Administrative Science Quarterly, 31, 78-108. https://doi.org/10.2307/2392767

Beane, M. (2019). Shadow learning: Building robotic surgical skill when approved means fail. Administrative Science Quarterly, 64, 87-123. https://doi.org/10.1177/0001839217 751692

Biggio, B., \& Roli, F. (2018). Wild patterns: Ten years after the rise of adversarial machine learning. Pattern Recognition, 84, 317-331. https://doi.org/10.1016/j.patcog.2018.07.023

Boulding, K. E. (1956). General systems theory-The skeleton of science. Management Science, 2, 197-208. https://doi.org/10.1287/mnsc.2.3.197

Brynjolfsson, E., Mitchell, T., \& Rock, D. (2018, May). What can machines learn, and what does it mean for occupations and the economy? AEA Papers and Proceedings, 108, 43-47.

Carlson, M. (2015). The robotic reporter: Automated journalism and the redefinition of labor, compositional forms, and journalistic authority. Digital Journalism, 3, 416-431. https://doi.org/10.1080/21670811.2014.976412

Chan, N. K., \& Humphreys, L. (2018). Mediatization of social space and the case of Uber drivers. Media and Communication, 6, 29-38. https://doi.org/10.17645/mac.v6i2.1316 
Christin, A. (2017). Algorithms in practice: Comparing web journalism and criminal justice. Big Data \& Society, 4, 1-14. https://doi.org/10.1177/2053951717718855

Chui, M., \& Malhotra, S. (2018). Notes from the AI frontier: AI adoption advances, but foundational barriers remain. https://www.mckinsey.com/featured-insights/artificialintelligence/ai-adoption-advances-but-foundational-barriers-remain

DeSanctis, G., \& Poole, M. S. (1994). Capturing the complexity in advanced technology use: Adaptive structuration theory. Organization Science, 5, 121-147. https://doi. org/10.1287/orsc.5.2.121

Diktonius, J. (2016, October 17). Tieto is the first Nordic company to appoint Artificial Intelligence to the leadership team of the new data-driven business unit. https://web.archive. org/web/20190919205734/https://www.tieto.com/en/newsroom/all-news-and-releases/ corporate-news/2016/10/tieto-the-first-nordic-company-to-appoint-artificial-intelligenceto-the-leadership-team-of-the-new-data-driven-business/

Duhigg, C. (2012, February 16). How companies learn your secrets. New York Times. Retrieved from https://www.nytimes.com/2012/02/19/magazine/shopping-habits.html ?pagewanted=1\&_r=1\&hp

Edwards, R. C. (1981). The social relations of production at the point of production. In Zey-Ferrell, M., \& Aiken, M. (Eds.). Complex organizations: Critical perspectives (pp. 156-182). Scott, Foresman.

Ekbia, H., \& Nardi, B. (2014). Heteromation and its (dis)contents: The invisible division of labor between humans and machines. First Monday, 19(6). https://doi.org/10.5210/ fm.v19i6.5331

Elliott, A. (2019). The culture of AI: Everyday life and the digital revolution. Routledge.

Emirbayer, M., \& Mische, A. (1998). What is agency? American Journal of Sociology, 103, 962-1023. https://www.jstor.org/stable/10.1086/231294

Faraj, S., Pachidi, S., \& Sayegh, K. (2018). Working and organizing in the age of the learning algorithm. Information and Organization, 28, 62-70. https://doi.org/10.1016/j.infoand org.2018.02.005

Foucault, M. (1979). Authorship: What is an author? Screen, 20(1), 13-34. https://doi. org/10.1093/screen/20.1.13

Fraune, M. R. (2020). Our robots, our team: Robot anthropomorphism moderates group effects in human-robot teams. Frontiers in Psychology, 11(1275), 1-14. https://doi. org/10.3389/fpsyg.2020.01275

Gambino, A., Fox, J., \& Ratan, R. A. (2020). Building a stronger CASA: Extending the computers are social actors paradigm. Human-Machine Communication, 1, 71-86. https:// doi.org/10.30658/hmc.1.5

Gaudiello, I., Zibetti, E., Lefort, S., Chetouani, M., \& Ivaldi, S. (2016). Trust as indicator of robot functional and social acceptance. An experimental study on user conformation to iCub answers. Computers in Human Behavior, 61, 633-655. https://doi.org/10.1016/j. chb.2016.03.057

Giddens, A. (1979). Central problems in social theory: Action, structure and contradiction in social analysis. University of California Press.

Giddens, A. (1984). The constitution of society. University of California Press. 
Graefe, A., Haim, M., Haarmann, B., \& Brosius, H.-B. (2018). Readers' perception of computer-generated news: Credibility, expertise, and readability. Journalism, 19, 595610. https://doi.org/10.1177/1464884916641269

Gray, M. L., \& Suri, S. (2019). Ghost work: How to stop Silicon Valley from building a new global underclass. Houghton Mifflin Harcourt.

Gunkel, D. J. (2012). Communication and artificial intelligence: Opportunities and challenges for the 21st Century. Communication +1, 1(1), Article 1. https://doi.org/10.7275/ R5QJ7F7R

Guzman, A. L. (2016). The messages of mute machines: Human-Machine Communication with industrial technologies. Communication +1, 5(1), Article 4. https://doi.org/10.7275/ R57P8WBW

Guzman, A. L. (Ed.). (2018). Human-Machine Communication: Rethinking communication, technology, and ourselves. Peter Lang Publishing, Inc.

Guzman, A. L. (2020). Ontological boundaries between humans and computers and the implications for Human-Machine Communication. Human-Machine Communication, 1, 37-54. https://doi.org/10.30658/hmc.1.3

Guzman, A. L., \& Lewis, S. C. (2020). Artificial intelligence and communication: A humanmachine communication research agenda. New Media \& Society, 22, 70-86. https://doi. org/10.1177/1461444819858691

Howard, L. A., \& Geist, P. (1995). The dialectic of control in a merging organization. Communication Monographs, 62, 110-131. https://doi.org/10.1080/03637759509376352

IBM Watson Talent. (2021). https://www.ibm.com/services/talent-management?mhsrc=ibmsearch_a\&mhq=Watson\%20Talent

Janlert, L. E., \& Stolterman, E. (2017). The meaning of interactivity: Some proposals for definitions and measures. Human-Computer Interaction, 32(3), 103-138. https://doi. org/10.1080/07370024.2016.1226139

Kellogg, K. C., Valentine, M. A., \& Christin, A. (2020). Algorithms at work: The new contested terrain of control. Academy of Management Annals, 14, 366-410. https://doi. org/10.5465/annals.2018.0174

Krizhevsky, A., Sutskever, I., \& Hinton, G. E. (2012). ImageNet classification with deep convolutional neural networks. Advances in Neural Information Processing Systems, 25, 1097-1105. https://doi.org/10.1145/3065386

Latour, B. (1991). Technology is society made durable. In J. Law (Ed.), A sociology of monsters. Essays on power, technology and domination (pp. 103-131). Routledge.

Lee, C., Rahafrooz, M., \& Lee, O. K. D. (2017). What are the concerns of using a ridesharing service? An investigation of Uber. In Proceeding of the 23rd Americas Conference on Information Systems (AMCIS 2017), 10-12 August, Boston, Massachusetts.

Leonardi, P. M. (2011). When flexible routines meet flexible technologies: Affordance, constraint, and the imbrication of human and material agencies. MIS Quarterly, 35, 147167. https://doi.org/10.2307/23043493

Levy, K. E. C. (2015). The contexts of control: Information, power, and truck-driving work. The Information Society, 31, 160-174. https://doi.org/10.1080/01972243.2015.998105

Lewis, S. C., Guzman, A. L., \& Schmidt, T. R. (2019). Automation, journalism, and humanmachine communication: Rethinking roles and relationships of humans and machines in news. Digital Journalism, 7, 409-427. https://doi.org/10.1080/21670811.2019.1577147 
Majchrzak, A., Rice, R., Malhotra, A., King, N., \& Ba, S. (2000). Technology adaptation: The case of a computer-supported inter-organizational virtual team. MIS Quarterly, 24, 569-600. https://doi.org/10.2307/3250948

Malone, T. W. (2018). Superminds: The surprising power of people and computers thinking together. Little, Brown, and Company.

McPhee, R. D., Poole, M. S., \& Iverson, J. (2014). Structuration theory. In L. L. Putnam \& D. K. Mumby (Eds.), The SAGE handbook of organizational communication: Advances in theory, research, and methods (3rd ed., pp. 75-100). Sage Publications.

Moore, A. (2019, June 7). When AI becomes an everyday technology. Harvard Business Review. https://hbr.org/2019/06/when-ai-becomes-an-everyday-technology

Neff, G., \& Nagy, P. (2016). Automation, algorithms, and politics| Talking to Bots: Symbiotic agency and the case of Tay. International Journal of Communication, 10, 4915-4931. https://ijoc.org/index.php/ijoc/article/view/6277

Newlands, G. (2020, online). Algorithmic surveillance in the gig economy: The organization of work through Lefebvrian conceived space. Organization Studies. https://doi. org/10.1177/0170840620937900

Noble, D. F. (2011). Forces of production: A social history of industrial automation. Transaction Publishers.

O’Neil, C. (2016). Weapons of math destruction: How big data increases inequality and threatens democracy. Broadway Books.

Orlikowski, W. J. (1992). The duality of technology: Rethinking the concept of technology in organizations. Organization Science, 3, 398-427. https://doi.org/10.1287/orsc.3.3.398

Orlikowski, W. J. (2007). Sociomaterial practices: Exploring technology at work. Organization Studies, 28, 1435-1448. https://doi.org/10.1177/0170840607081138

Orlikowski, W. J., \& Scott, S. V. (2008). Sociomateriality: Challenging the separation of technology, work and organization. Academy of Management Annals, 2, 433-474. https:// doi.org/10.5465/19416520802211644

Orlikowski, W. J., \& Yates, J. (1994). Genre repertoire: The structuring of communicative practices in organizations. Administrative Science Quarterly, 39, 541-574. https://doi. org/10.2307/2393771

Perc, M., Ozer, M., \& Hojnik, J. (2019). Social and juristic challenges of artificial intelligence. Palgrave Communications, 5, 1-7. https://doi.org/10.1057/s41599-019-0278-x

Pondy, L. R., \& Mitroff, I. I. (1979). Beyond open systems models of organizations. In B. M. Staw (Ed.), Research in Organization Behavior (vol. 1, pp. 3-39). JAI Press.

Pozzebon, M., \& Pinsonneault, A. (2005). Challenges in conducting empirical work using structuration theory: Learning from IT research. Organization Studies, 26, 1353-1376. https://doi.org/10.1177/0170840605054621

Raghuram, S., Hill, N. S., Gibbs, J. L., \& Maruping, L. M. (2019). Virtual work: Bridging research clusters. Academy of Management Annals, 13, 308-341. https://doi.org/10.5465/ annals.2017.0020

Reeves, B., \& Nass, C. (1996). Media equation: How people treat computers, television, and new media like real people and places. Cambridge University Press.

Riley, P. (1983). A structurationist account of political culture. Administrative Science Quarterly, 28(3), 414-437. https://doi.org/10.2307/2392250 
Rosenblat, A. (2019). Uberland: How algorithms are rewriting the rules of work. University of California Press.

Rosenblat, A., \& Stark, L. (2016). Algorithmic labor and information asymmetries: A case study of Uber's drivers. International Journal of Communication, 10, 3758-3784. https:// doi.org/10.2139/ssrn.2686227

Russell, S., \& Norvig, P. (2019). Artificial intelligence: A modern approach (4th ed.). Pearson Education. https://doi.org/10.1017/S0269888900007724

Sparrow, B., Liu, J., \& Wegner, D. M. (2011). Google effects on memory: Cognitive consequences of having information at our fingertips. Science, 333, 776-778. https://doi. org/10.1126/science.1207745

Stillman, L., \& Stoecker, R. (2005). Structuration, ICTs, and community work. The Journal of Community Informatics, 1(3), 83-102.

Sturken, M., Thomas, D., \& Ball-Rokeach, S. J. (Eds.). (2004). Technological visions: The hopes and fears that shape new technologies. Temple University Press.

Suchman, L. A. (2007). Human-machine reconfigurations: Plans and situated actions (2nd ed.) Cambridge University Press.

Sundar, S. S., Bellur, S., Oh, J., Jia, H., \& Kim, H.-S. (2016). Theoretical importance of contingency in human-computer interaction: Effects of message interactivity on user engagement. Communication Research, 43, 595-625. https://doi.org/10.1177/0093650214534962

Sundararajan, A. (2016). The sharing economy: The end of employment and the rise of crowdbased capitalism. The MIT Press. https://doi.org/10.2307/j.ctt1c2cqh3

Tompkins, P. K., \& Cheney, G. (1985). Communication and unobtrusive control in contemporary organizations. In R. D. McPhee \& P. K. Tompkins (Eds.), Organizational communication: Traditional themes and new directions (pp. 179-210). Sage Publications.

Waytz, A., Heafner, J., \& Epley, N. (2014). The mind in the machine: Anthropomorphism increases trust in an autonomous vehicle. Journal of Experimental Social Psychology, 52, 113-117. https://doi.org/10.1016/j.jesp.2014.01.005

Weber, M. S., \& Kosterich, A. (2018). Coding the news: The role of computer code in filtering and distributing news. Digital Journalism, 6, 310-329.

West, D. M., \& Allen, J. R. (2018, April 24). How artificial intelligence is transforming the world. Brookings. https://www.brookings.edu/research/how-artificial-intelligence-istransforming-the-world/

Wiener, N. (1950). The human use of human beings: Cybernetics and society. Houghton Mifflin.

Wood, A. J., Graham, M., Lehdonvirta, V., \& Hjorth, I. (2019). Good gig, bad gig: Autonomy and algorithmic control in the global gig economy. Work, Employment, \& Society, 33, 56-75. https://doi.org/10.1177/0950017018785616

Yan, B., Lewis, K., Figge, P., Hollingshead, A., Alexander, K. S., Kim, Y. J., \& Fang, C. (2020). Intelligent machines and teamwork: Help or hindrance? In Academy of Management Proceedings (Vol. 2020, No. 1, p. 21962). Briarcliff Manor, NY 10510: Academy of Management.

Zuboff, S. (2019). The age of surveillance capitalism: The fight for a human future at the new frontier of power. Public Affairs. 

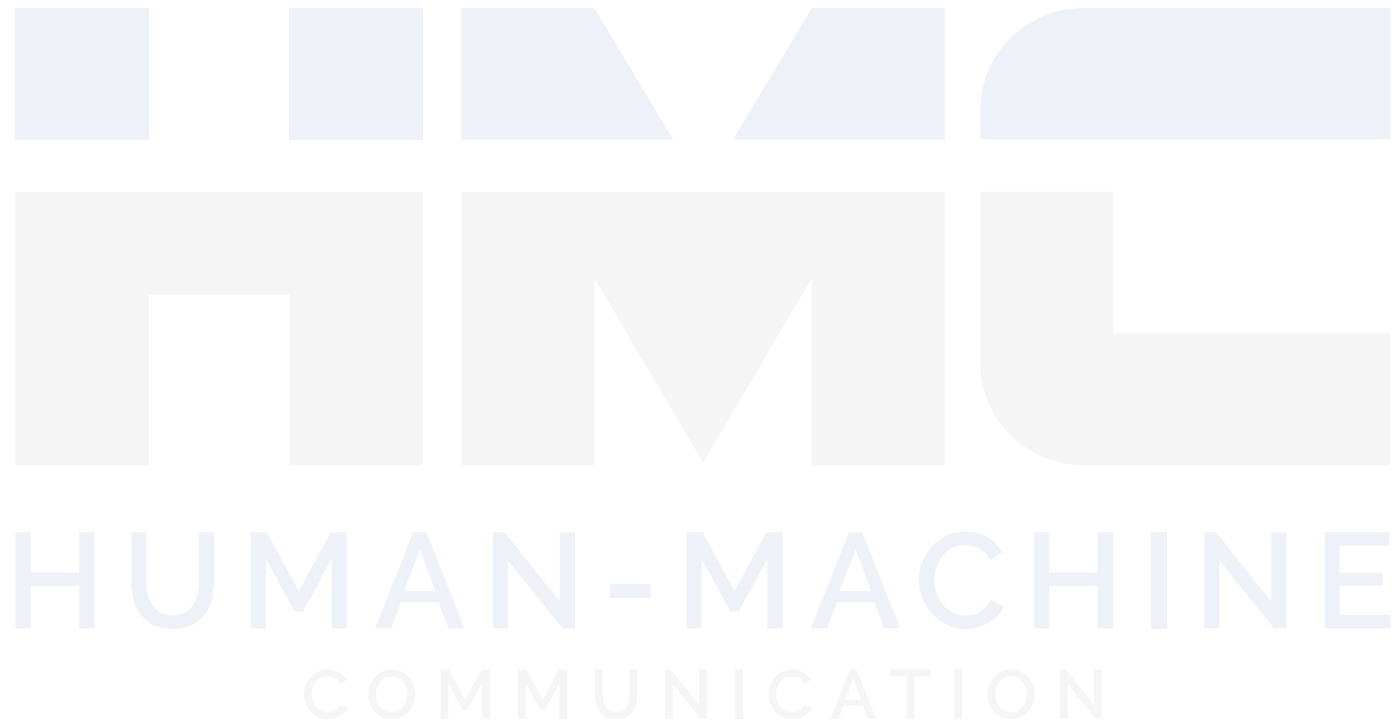